\title{
On a sum of squares of integers in arithmetic progression
}

Abdullah-Al-Kafi Majumdar*

Beppu-shi Oaza Tsurumi, Renace Beppu, Beppu-shi, Japan

\begin{tabular}{|c|c|}
\hline ARTICLE INFO & ABSTRACT \\
\hline Article History & This paper derives the conditions under which the sum \\
\hline Received: 19 September 2021 & of squares of $(2 N+1)$ natural numbers in the arithmetic \\
\hline Revised: 30 December 2021 & progression is a perfect square. It is shown that the \\
\hline Accepted: 30 December 2021 & problem leads to a Diophantine equation, which in turn \\
\hline $\begin{array}{l}\text { Keywords: Sum of squares, gcd, Arithmetic } \\
\text { progression, Diophantine equation }\end{array}$ & $\begin{array}{l}\text { indicates that there is, in fact, an infinite number of such } \\
\text { numbers. Some particular cases are investigated. }\end{array}$ \\
\hline
\end{tabular}

\section{Introduction}

It is well-known that, for any integer $n(\geq 1)$,

$$
1^{3}+2^{3}+3^{3}+\ldots+n^{3}=\left[\frac{n(n+1)}{2}\right]^{2}
$$

which expresses the interesting property that the sum of cubes of the first $n$ (positive) integers is a perfect square. This paper finds the condition that the sum of squares of $(2 N+1)$ integers in the arithmetic progression is a square. It is found that the problem reduces to that of solving a Diophantine equation.

Sastry (1992) raised the problem of characterizing the arithmetic progression of natural numbers of length 49, whose sum of squares is also a square. This paper generalizes the problem of Sastry (1992) to the case of numbers in the arithmetic progression of length $(2 N+1)$. This is done in Section 3. Some background materials related to the problem are given in the next section. Section 4 deals with some particular cases. The paper is concluded with some remarks in Section 5.

\section{Background Material}

This section gives some results that would be needed later in Section 3. In Section 3, it is shown that the problem of finding a sequence of $(2 N+1)$ (positive) integers in arithmetic progression whose sum of squares is itself a square reduces to the problem of solving a Diophantine equation of the form $a^{2}=b^{2}+m c^{2}$, where $m(>0)$ is an integer. In solving the equation, note that, if $a=a_{0}, b=b_{0}$ and $c=c_{0}$ is a solution, so also is $a=s a_{0}, b=s b_{0}, c=s c_{0}$ for any integer $s>0$. It is thus sufficient to find the primitive solution of the equation. The lemma below gives the solution of the Diophantine equation $a^{2}=b^{2}+2 c^{2}$.

Lemma 2.1: Consider the Diophantine equation

$$
a^{2}=b^{2}+2 c^{2}, \operatorname{gcd}(a, b, c)=1 \text {. }
$$

The (positive) primitive solutions of the above equation are

$$
a=\alpha^{2}+2 \beta^{2}, b=\left|\alpha^{2}-2 \beta^{2}\right|, c=2 \alpha \beta,
$$

where $\alpha$ and $\beta$ are (positive) integers with $\operatorname{gcd}(\alpha, \beta)$ $=1$ and $\alpha$ is odd.

Proof: Clearly, any $b$ satisfying the equation (2.1) must be odd, which in turn implies that $a$ is odd and $c$ is even. It then follows that $\alpha$ in (2.2) must be odd. Now, with $a, b, c$ given in (2.2),

$$
a^{2}=\left(\alpha^{2}+2 \beta^{2}\right)^{2}=\left(\alpha^{2}-2 \beta^{2}\right)^{2}+8 \alpha^{2} \beta^{2}=b^{2}+2 c^{2},
$$

which shows that (2.2) is indeed a solution of the equation (2.1).

Lemma 2.2: Consider the Diophantine equation

$$
a^{2}=b^{2}+p c^{2}, \operatorname{gcd}(a, b, c)=1,
$$

where $p(\geq 3)$ is a prime. A set of primitive solutions of the equation (2.3) are 


$$
a=\alpha+p \beta^{2}, b=\left|\alpha-p \beta^{2}\right|, c=2 \alpha \beta,
$$

where $\alpha$ and $\beta$ are (positive) integers with $\alpha \neq \beta$, $\operatorname{gcd}(\alpha, \beta)=1, \alpha$ and $\beta$ are of opposite parity (that is, one of $\alpha$ and $\beta$ is odd, and the other one is even), and $\alpha$ is not a multiple of $p$.

By direct substitution, it may be verified that (2.4) constitutes a solution of the Diophantine equation (2.3). When both $\alpha$ and $\beta$ are odd, $a, b$ and $c$ (in (2.4)) is even. Thus, for the primitive solution, $\alpha$ and $\beta$ must be of opposite parity. It may be mentioned here that Dickson (1971) gives the following solution of the equation (1.3):

$$
a=\lambda\left(p t^{2}+1\right), b=\lambda\left(p t^{2}-1\right), c=2 \lambda t,
$$

where $\lambda$ and $t$ are any positive integers. It may be remarked here that, (2.5) does not constitute the general solution of (2.1). For example, when $p=2$, by Lemma 2.1 with $\alpha=3, \beta=1,(11,7,6)$ is a solution of (2.1), which cannot be obtained from (2.5) for any integral values of $\lambda$ and $t$. Vowe (1993), in his solution to the problem of Sastry (1992), uses a more general solution. It may be mentioned here that (2.4) does not give all the solutions of the equation (2.3); for example, for $p(\geq 3)$, it may readily be verified that $a=\frac{p+1}{2}, b=\frac{p-1}{2}, c=1$ is a primitive solution; however, Lemma 2.2 is sufficient for this paper.

A particular case of Lemma 2.2 is one when $p=3$; this case has been studied, to some extent, by Majumdar (2018, 2020). The following lemma gives two sets of solutions of the Diophantine equation $a^{2}=b^{2}+p q c^{2}$, where $p$ and $q$ are two distinct odd primes.

Lemma 2.3: Consider the Diophantine equation

$$
a^{2}=b^{2}+p q c^{2}, \operatorname{gcd}(a, b, c)=1,
$$

where $p$ and $q(>p \geq 3)$ are primes. Then, its two sets of (positive) primitive solutions are as follows:

(1) $a=p \alpha^{2}+q \beta^{2}, b=\left|p \alpha^{2}-q \beta^{2}\right|, c=2 \alpha \beta$, where $\alpha$ and $\beta$ are (positive) integers with $\alpha \neq \beta$, $\operatorname{gcd}(\alpha, \beta)=1, \alpha$ and $\beta$ are of opposite parity, and $\alpha$ is not a multiple of $q, \beta$ is not a multiple of $p$,

(2) $a=v^{2}+p q \theta^{2}, b=\left|v^{2}-p q \theta^{2}\right|, c=2 v \theta$,

where $v$ and $\theta$ are (positive) integers with $\operatorname{gcd}$ $(v, \theta)=1$ and both $v$ and $\theta$ are of opposite parity, and $v$ is not a multiple of $p$ or $q$.

When $p=2$, both the parameters $\beta$ and $v$ are odd.

Proof: With $a, b, c$ given by (2.7),

$$
\begin{aligned}
a^{2} & =\left(p \alpha^{2}+q \beta^{2}\right)^{2}=\left(p \alpha^{2}-q \beta^{2}\right)^{2}+4 p q \alpha^{2} \beta^{2} \\
& =b^{2}+p q c^{2} ;
\end{aligned}
$$

also, from (2.8),

$$
\begin{aligned}
a^{2} & =\left(v^{2}+p q \theta^{2}\right)^{2}=\left(v^{2}-p q \theta^{2}\right)^{2}+4 p q v^{2} \theta^{2} \\
& =b^{2}+p q c^{2} .
\end{aligned}
$$

Thus, each of (2.7) and (2.8) forms a solution of the Diophantine equation (2.6).

Now, replacing $v$ by $p v$ in (2.8), one gets the solution

$$
a=p\left(p v^{2}+q \theta^{2}\right), b=p\left|p v^{2}-q \theta^{2}\right|, c=2 p v \theta,
$$

which is just $p$ times the solution (2.7).

When $p(\geq 3)$ is odd, so that $a, b$ and $c$ are pairwise co-prime, the parameters $\alpha$ and $\beta$ in part (1) of the lemma must be of opposite parity, while for $p=2$, $\beta$ must be odd. Again, when $p$ is odd, the parameters $v$ and $\theta$ in part (2) are of opposite parity, and $v$ is odd when $p=2$.

Extension of Lemma 2.3 is the following one.

Lemma 2.4: Consider the Diophantine equation

$$
a^{2}=b^{2}+p q r c^{2}, \operatorname{gcd}(a, b, c)=1,
$$

where $p, q$ and $r(r>q>p \geq 3)$ are three distinct primes. Then, its four sets of (positive) primitive solutions are given below, where in each case, the parameters $\alpha$ and $\beta$ are (positive) integers with $\alpha \neq \beta$, $\operatorname{gcd}(\alpha, \beta)=1$, and $\alpha$ and $\beta$ are of opposite parity :

(1) $a=p \alpha^{2}+q r \beta^{2}, b=\left|p \alpha^{2}-q r \beta^{2}\right|, c=2 \alpha \beta$,

where $\alpha$ is not a multiple of $q$ or $r, \beta$ is not a multiple of $p$, 
(2) $a=q \alpha^{2}+p r \beta^{2}, b=\left|q \alpha^{2}-p r \beta^{2}\right|, c=2 \alpha \beta$, where $\alpha$ is not a multiple of $p$ or $r, \beta$ is not a multiple of $q$,

(3) $a=r \alpha^{2}+p q \beta^{2}, b=\left|r \alpha^{2}-p q \beta^{2}\right|, c=2 \alpha \beta$, where $\alpha$ is not a multiple of $p$ or $q, \beta$ is not a multiple of $r$,

(4) $a=\alpha^{2}+p q r \beta^{2}, b=\left|\alpha^{2}-p q r \beta^{2}\right|, c=2 \alpha \beta$, $\alpha$ is not a multiple of $p$ or $q$ or $r$.

When $p=2$, the parameter $\beta$ in part (1) is odd, and the parameter $\alpha$ must be odd in each of the remaining three cases.

Proof: The proof follows from the following identities :

$$
\begin{aligned}
& \left(p \alpha^{2}+q r \beta^{2}\right)^{2}=\left(p \alpha^{2}-q r \beta^{2}\right)^{2}+4 p q r \alpha^{2} \beta^{2} ; \\
& \left(q \alpha^{2}+p r \beta^{2}\right)^{2}=\left(q \alpha^{2}-p r \beta^{2}\right)^{2}+4 p q r \alpha^{2} \beta^{2} ; \\
& \left(r \alpha^{2}+p q \beta^{2}\right)^{2}=\left(r \alpha^{2}-p q \beta^{2}\right)^{2}+4 p q r \alpha^{2} \beta^{2} ; \\
& \left(\alpha^{2}+p q r \beta^{2}\right)^{2}=\left(\alpha^{2}-p q r \beta^{2}\right)^{2}+4 p q r \alpha^{2} \beta^{2} .
\end{aligned}
$$

The $a, b$ and $c$ in (2.9) are pair-wise relatively prime; $\alpha$ and $\beta$ both cannot be even; moreover $\alpha$ and $\beta$ both cannot be odd when $p(\geq 3)$ is odd. Also, when $p=2$, so that $a, b$ and $c$ are pairwise co-prime, the conditions mentioned in the lemma must be satisfied.

The lemma below extends to the case involving four distinct primes.

Lemma 2.5: Consider the Diophantine equation

$$
a^{2}=b^{2}+p q r s c^{2}, \operatorname{gcd}(a, b, c)=1,
$$

where $p, q, r$ and $s(s>r>q>p \geq 3)$ are four primes. Then, its eight sets of (positive) primitive solutions are as listed below, where in each case, $\alpha$ and $\beta$ are (positive) integers with $\alpha \neq \beta, \operatorname{gcd}(\alpha, \beta)=1, \alpha$ and $\beta$ are of opposite parity :

(1) $a=p \alpha^{2}+q r s \beta^{2}, b=\left|p \alpha^{2}-q r s \beta^{2}\right|, c=2 \alpha \beta$, where $\alpha$ is not a multiple of $q$ or $r$ or $s, \beta$ is not a multiple of $p$,

(2) $a=q \alpha^{2}+p r s \beta^{2}, b=\left|q \alpha^{2}-p r s \beta^{2}\right|, c=2 \alpha \beta$, where $\alpha$ is not a multiple of $p$ or $r$ or $s, \beta$ is not a multiple of $q$,

(3) $a=r \alpha^{2}+p q s \beta^{2}, b=\left|r \alpha^{2}-p q s \beta^{2}\right|, c=2 \alpha \beta$, where $\alpha$ is not a multiple of $p$ or $q$ or $s, \beta$ is not a multiple of $r$,

(4) $a=s \alpha^{2}+p q r \beta^{2}, b=\left|s \alpha^{2}-p q r \beta^{2}\right|, c=2 \alpha \beta$,

where $\alpha$ is not a multiple of $p$ or $q$ or $r, \beta$ is not a multiple of $s$,

(5) $a=p q \alpha^{2}+r s \beta^{2}, b=\left|p q \alpha^{2}-r s \beta^{2}\right|, c=2 \alpha \beta$,

where $\alpha$ is not a multiple of $r$ or $s, \beta$ is not a multiple of $p$ or $q$,

(6) $a=p r \alpha^{2}+q s \beta^{2}, b=\left|p r \alpha^{2}-q s \beta^{2}\right|, c=2 \alpha \beta$,

where $\alpha$ is not a multiple of $q$ or $s, \beta$ is not a multiple of $p$ or $r$,

(7) $a=p s \alpha^{2}+q r \beta^{2}, b=\left|p s \alpha^{2}-q r \beta^{2}\right|, c=2 \alpha \beta$,

where $\alpha$ is not a multiple of $q$ or $r, \beta$ is not a multiple of $p$ or $s$,

(8) $a=\alpha^{2}+p q r s \beta^{2}, b=\left|\alpha^{2}-p q r s \beta^{2}\right|, c=2 \alpha \beta$,

where $\alpha$ is not a multiple of $p$ or $q$ or $r$ or $s$.

When $p=2$, the parameter $\beta$ in each of part (1), part (5), part (6), and part (7) is odd, while $\alpha$ is odd in each of the remaining cases.

Proof: The proof follows from the following eight identities :

$$
\begin{aligned}
& \left(p \alpha^{2}+q r s \beta^{2}\right)^{2}=\left(p \alpha^{2}-q r s \beta^{2}\right)^{2}+4 p q r s \alpha^{2} \beta^{2} ; \\
& \left(q \alpha^{2}+p r s \beta^{2}\right)^{2}=\left(q \alpha^{2}-p r s \beta^{2}\right)^{2}+4 p q r s \alpha^{2} \beta^{2} ; \\
& \left(r \alpha^{2}+p q s \beta^{2}\right)^{2}=\left(r \alpha^{2}-p q s \beta^{2}\right)^{2}+4 p q r s \alpha^{2} \beta^{2} ; \\
& \left(s \alpha^{2}+p q r \beta^{2}\right)^{2}=\left(s \alpha^{2}-p q r \beta^{2}\right)^{2}+4 p q r s \alpha^{2} \beta^{2} . \\
& \left(p q \alpha^{2}+r s \beta^{2}\right)^{2}=\left(p q \alpha^{2}-r s \beta^{2}\right)^{2}+4 p q r s \alpha^{2} \beta^{2} ; \\
& \left(p r \alpha^{2}+q s \beta^{2}\right)^{2}=\left(p r \alpha^{2}-q s \beta^{2}\right)^{2}+4 p q r s \alpha^{2} \beta^{2} ; \\
& \left(p s \alpha^{2}+q r \beta^{2}\right)^{2}=\left(p s \alpha^{2}-q r \beta^{2}\right)^{2}+4 p q r s \alpha^{2} \beta^{2} ; \\
& \left(\alpha^{2}+p q r s \beta^{2}\right)^{2}=\left(\alpha^{2}-p q r s \beta^{2}\right)^{2}+4 p q r s \alpha^{2} \beta^{2} .
\end{aligned}
$$

Clearly, when $p(\geq 3)$, in order that $a, b$ and $c$ are pair-wise relatively prime, the parameters $\alpha$ and $\beta$ cannot be both or both even. It is evident that, when $p=2$, the conditions in the lemma are satisfied.

\section{The problem}

Consider the following sequence of $(2 N+1)$ (positive) integers in arithmetic progression :

$$
y-N d, y-(N-1) d, \ldots, y-d, y, y+d, \ldots, y+N d,
$$


where $d(>0)$ is the common difference and $y>N d$. Note that the latter condition guarantees that all the terms of the sequence are positive.

Let $S S(N)$ be the sum of squares of the terms of the sequence, that is,

$$
S S(N)=y^{2}+\sum_{i=1}^{N}\left[(y-i d)^{2}+(y+i d)^{2}\right]
$$

Then, $S S(N)$ may be expressed as follows.

Lemma 3.1: For all $N \geq 1$,

$$
S S(N)=(2 N+1)\left[y^{2}+\frac{N(N+1)}{3} d^{2}\right], y>N d .
$$

Proof: From (3.1), after simplification,

$$
S S(N)=(2 N+1) y^{2}+2 d^{2} \sum_{i=1}^{N} i^{2}
$$

Since $\sum_{i=1}^{N} i^{2}=\frac{N(N+1)(2 N+1)}{6}$, plugging in (i), the desired result is obtained after some simple algebraic manipulation.

The problem is to find the condition such that $S S(N)$, given in Lemma 3.1, is a perfect square. To simplify the situation, the following assumptions are made :

Assumption 3.1: Let the integer $N(>1)$ be such that $2 N+1$ is a square.

Assumption 3.2: One of $N$ and $N+1$ is divisible by 3 .

Assumption 3.2 is meant to guarantee that $\frac{N(N+1)}{3}$ is an integer. Then, the following result can be proved.

Lemma 3.2: Let the integer $N$ be such that $2 N+1=M^{2}$ for some integer $M(>0)$.

Then, $N$ is a multiple of 4 ; furthermore, under Assumption 3.2, $N$ is a multiple of 3 .

Proof: Since $2 N=(M-1)(M+1)$, it follows that each of $M-1$ and $M+1$ is even. Now, since one of $M-1$ and $M+1$ is of the form $4 m$ and the other one is of form $4 m+2$, it follows that $N$ is a multiple of 4 . Now, writing

$$
M^{2}=N+(N+1),
$$

it follows that, under Assumption 3.2, $M$ is not divisible by 3 . Then one of $M-1$ and $M+1$ is divisible by 3 . Consequently, $N$ is divisible by 3 .
The following lemma gives the main result of this paper.

Lemma 3.3: Let $N$ be such that

$2 N+1=M^{2}$ for some integer $M>0$.

Then, $S S(N)$ is a perfect square if and only if $y^{2}+\frac{N(N+1)}{3} d^{2}=x^{2}$ for some integer $x$.

Proof: is evident from Lemma 3.1.

\section{Some particular cases}

Section 3 derives the condition that $S S(N)$, given by (3.2), is a perfect square under Assumption 3.1 and Assumption 3.2. Lemma 3.2 shows that, under these two assumptions, $N$ is a multiple of 12. In this section, some particular cases are studied. By Assumption 3.1, $(2 N+1)$ is a square. The following table gives such $N$ and the related information, when $N<1000$.

Table 1. Values of $N$ for which $2 N+1$ is a square

\begin{tabular}{ccl}
\hline $\mathrm{N}$ & $2 N+1$ & $\frac{N(N+1)}{3}$ \\
\hline 12 & $5^{2}$ & $2^{2} \times 13$ \\
24 & $7^{2}$ & $2 \times 10^{2}$ \\
60 & $11^{2}$ & $2^{2} \times 5 \times 61$ \\
84 & $13^{2}$ & $2^{2} \times 5 \times 7 \times 17$ \\
144 & $17^{2}$ & $3 \times 4^{2} \times 5 \times 29$ \\
180 & $19^{2}$ & $2^{2} \times 3 \times 5 \times 181$ \\
264 & $23^{2}$ & $2^{3} \times 5 \times 11 \times 53$ \\
312 & $25^{2}$ & $2^{2} \times 13 \times 313$ \\
420 & $29^{2}$ & $2^{2} \times 5 \times 7 \times 421$ \\
480 & $31^{2}$ & $2^{5} \times 5 \times 13 \times 37$ \\
612 & $35^{2}$ & $2^{2} \times 3 \times 17 \times 613$ \\
684 & $37^{2}$ & $2^{2} \times 3 \times 5 \times 19 \times 137$ \\
840 & $41^{2}$ & $2^{3} \times 5 \times 7 \times 29^{2}$ \\
\hline
\end{tabular}

In the following five examples, the cases $N=12,24$, $60,84,264$ are considered in detail.

Example 4.1: When $N=12$. In this case, by (3.2),

$$
S S(12)=5^{2}\left[y^{2}+13(2 d)^{2}\right] \text {. }
$$

Assuming that $S S(12)=(5 x)^{2}$ for some integer $x(>0)$, by Lemma 2.2 with $p=13$,

$$
y=13 \beta^{2}-\alpha^{2}, d=\alpha \beta \text {, }
$$


where $\alpha$ and $\beta$ are integers with $\operatorname{gcd}(\alpha, \beta)=1$, and $y>12 d=12 \alpha \beta$. It can be proved that $\beta>\alpha$, for otherwise,

$$
\beta \leq \alpha \Rightarrow y \equiv 13 \beta^{2}-\alpha^{2} \leq 12 \beta^{2} \leq 12 \alpha \beta,
$$

which leads to a contradiction.

When $\alpha=1$, the minimum admissible value of $\beta$ is $\beta=2$. When $\alpha=1, \beta=2$ in (4.1), then $\quad d=2$ and $y=$ 51. This gives the series

$$
27^{2}+29^{2}+31^{2}+\ldots+75^{2}=(5 \times 53)^{2} .
$$

Letting $\alpha=1$ and $\beta=3,4$ in succession, the following series result :

$$
\begin{aligned}
& 80^{2}+83^{2}+\ldots+152^{2}=(5 \times 118)^{2} . \\
& 159^{2}+163^{2}+\ldots+255^{2}=(5 \times 209)^{2} .
\end{aligned}
$$

Again, putting $\alpha=2, \beta=3$, the following series is obtained :

$$
41^{2}+47^{2}+\ldots+185^{2}=(5 \times 121)^{2} .
$$

When $\alpha=3, \beta=4$, one gets the series

$$
55^{2}+67^{2}+\ldots+343^{2}=(5 \times 217)^{2},
$$

while, $\alpha=3, \beta=5$ gives the series

$$
136^{2}+151^{2}+\ldots+496^{2}=(5 \times 334)^{2} .
$$

The above examples show that to find primitive solutions of the Diophantine equation (4.1), it is unnecessary. that $\alpha$ and $\beta$ must be of the same parity; there is such a solution when $\alpha$ and $\beta$ are both odd.

Example 4.2: By (3.2) with $N=24$,

$$
S S(24)=7^{2}\left[y^{2}+2(10 d)^{2}\right] \text {. }
$$

Assuming that $S S(24)=(7 x)^{2}$ for some integer $x(>0)$, by Lemma 2.1,

$$
y=2 \beta^{2}-\alpha^{2}, 5 d=\alpha \beta,
$$

where $\alpha$ and $\beta$ are integers with $\operatorname{gcd}(\alpha, \beta)=1$. In this case, in order that $d$ is an integer, one of $\alpha$ and $\beta$ must be divisible by 5 . Also,

$$
\begin{gathered}
y \equiv 2 \beta^{2}-\alpha^{2}>24 d=\frac{24 \alpha \beta}{5} \\
\text { i.e., }\left(\beta-\frac{6}{5} \alpha\right)^{2}>\frac{97}{50} \alpha^{2} \\
\text { i.e., } \beta>\frac{6+\sqrt{\frac{97}{2}}}{5} \alpha=2.592839 \alpha .
\end{gathered}
$$

Thus, $\alpha$ and $\beta$ must satisfy the above inequality in this case.

Thus, when $\beta=5$, the only admissible value of $\alpha$ is $\alpha=1$, resulting in the series

$$
25^{2}+26^{2}+\ldots+73^{2}=(7 \times 51)^{2} .
$$

With $\beta=10$, the only two admissible values of $\alpha$ are $\alpha=1,3$, giving respectively the following series :

$$
\begin{aligned}
& 151^{2}+153^{2}+\ldots+247^{2}=(7 \times 201)^{2}, \\
& 47^{2}+53^{2}+\ldots+335^{2}=(7 \times 209)^{2} .
\end{aligned}
$$

When $\beta=13$, the only admissible $\alpha$ is $\alpha=5$, giving the series below :

$$
1^{2}+14^{2}+27^{2}+\ldots+625^{2}=(7 \times 363)^{2} .
$$

Also, $\beta=14, \alpha=5$ gives the series

$$
31^{2}+45^{2}+\ldots+703^{2}=(7 \times 417)^{2} .
$$

With $\alpha=10, \beta=27$, the series obtained is

$$
62^{2}+116^{2}+\ldots+2654^{2}=(7 \times 1558)^{2} \text {, }
$$

which reduces to

$$
31^{2}+58^{2}+\ldots+1327^{2}=(7 \times 779)^{2} .
$$

It may be mentioned here that the equation $2 \beta^{2}-\alpha^{2}$ $=31$ has the unique solution $\alpha=1, \quad \beta=4$, which is not admissible by the condition of the problem. Thus, unlike Lemma 2.1, $\alpha$ is not restricted to odd values only.

This may be recalled as the problem proposed by Sastry (1992). Vowe (2007) uses the solution

$$
y=\chi\left(2 \beta^{2}-\alpha^{2}\right), 5 d=\alpha \beta \gamma,
$$

where $\gamma(>0)$ is an integer. If the above solution is used, then $\gamma$ may be taken as an integer of the form $5 m$, solutions may be obtained by assigning admissible values to $\alpha$ and $\beta$.

Example 4.3: Letting $N=60$ in (3.2), one gets $S S(60)$ $=11^{2}\left[y^{2}+305(2 d)^{2}\right]$.

Assuming that $S S(60)=(11 x)^{2}$ for some integer $x$ (> 0 ), by Lemma 2.3 with $p=5, q=61$, one solution is

$$
y=61 \beta^{2}-5 \alpha^{2}, d=\alpha \beta,
$$

where $\alpha$ and $\beta$ are integers with $\operatorname{gcd}(\alpha, \beta)=1, \alpha$ is not a multiple of 61 and $\beta$ is not a multiple of 5 . Furthermore, the following condition must be satisfied:

$$
y \equiv 61 \beta^{2}-5 \alpha^{2}>60 d=60 \alpha \beta
$$

i.e., $\left(\beta-\frac{30}{61} \alpha\right)^{2}>\frac{1205}{61^{2}} \alpha^{2}$

$$
\text { i.e., } \beta>\frac{30+\sqrt{1205}}{61} \alpha=1.0608706 \alpha \text {. }
$$

When $\alpha=1$, then $\beta \geq 2$. Letting $\alpha=1, \beta=2$ in (4.3), one has $d=2$ and $y=239$. This case gives the series 


$$
119^{2}+121^{2}+\ldots+359^{2}=(11 \times 249)^{2} .
$$

With $\alpha=1, \beta=3$, the resulting series is

$$
364^{2}+367^{2}+\ldots+724^{2}=(11 \times 554)^{2} .
$$

Again, letting $\alpha=2, \beta=3$, the series obtained is

$$
169^{2}+175^{2}+\ldots+889^{2}=(11 \times 569)^{2} .
$$

When $\alpha=17, \beta=19$, the resulting series is

$$
1196^{2}+1519^{2}+\ldots+39956^{2}=(11 \times 23466)^{2} .
$$

Note that, to get primitive solutions of the Diophantine equation (4.3), the parameters $\alpha$ and $\beta$ need not be of opposite parity, contrary to Lemma 2.3.

By Lemma 2.3, the second solution is

$$
y=305 \beta^{2}-\alpha^{2}, d=\alpha \beta,
$$

where $\alpha$ and $\beta$ are integers with $\operatorname{gcd}(\alpha, \beta)=1, \alpha$ is not a multiple of 5 or 61 . Moreover,

$$
\begin{gathered}
y \equiv 305 \beta^{2}-\alpha^{2}>60 d=60 \alpha \beta \\
\text { i.e., }\left(\beta-\frac{30}{305} \alpha\right)^{2}>\frac{1205}{305^{2}} \alpha^{2} \\
\text { i.e., } \beta>\frac{30+\sqrt{1205}}{305} \alpha=0.202174 \alpha .
\end{gathered}
$$

Thus, in this case, $\alpha$ and $\beta$ satisfy the above inequality.

When $\alpha=1, \beta=1$ in (4.4), $d=1$ and $y=304$. This case gives the series

$$
244^{2}+245^{2}+\ldots+364^{2}=(11 \times 306)^{2} .
$$

When $\alpha=2, \beta=1$, the resulting series is

$$
181^{2}+183^{2}+\ldots+421^{2}=(11 \times 309)^{2},
$$

when $\alpha=3, \beta=1$, the series obtained is

$$
116^{2}+119^{2}+\ldots+376^{2}=(11 \times 314)^{2},
$$

and when $\alpha=4, \beta=1$, the series obtained is

$$
49^{2}+53^{2}+\ldots+529^{2}=(11 \times 321)^{2} .
$$

When $\beta=2$, the only admissible values of $\alpha$ are $\alpha=$ $1,3,7,9$, and the corresponding series are

$$
\begin{aligned}
& 1099^{2}+1101^{2}+\ldots+1339^{2}=(11 \times 1221)^{2}, \\
& 851^{2}+857^{2}+\ldots+1571^{2}=(11 \times 1229)^{2}, \\
& 331^{2}+345^{2}+\ldots+2011^{2}=(11 \times 1269)^{2}, \\
& 59^{2}+77^{2}+\ldots+2219^{2}=(11 \times 1301)^{2} .
\end{aligned}
$$

When $\alpha=9$, the minimum attainable value of $\beta$ is $\beta=2$, and the corresponding series is

$$
59^{2}+77^{2}+\ldots+2219^{2}=(11 \times 1301)^{2} .
$$

Note that, unlike Lemma 2.3, the parameters $\alpha$ and $\beta$ may be odd, as demonstrated above.

Example 4.4: (3.2) with $N=84$ gives

$$
S S(84)=13^{2}\left[y^{2}+595(2 d)^{2}\right] \text {. }
$$

Assuming that $S S(84)=(13 x)^{2}$ for some integer $x(>0)$, by Lemma 2.4 with $p=5, q=7, r=17$, one solution is

$$
y=119 \beta^{2}-5 \alpha^{2}, d=\alpha \beta,
$$

where $\alpha$ and $\beta$ are integers with $\operatorname{gcd}(\alpha, \beta)=1, \alpha$ is not a multiple of 7 or 17 , and $\beta$ is not a multiple of 5 . Also, $\alpha$ and $\beta$ satisfy the condition below.

$$
\begin{aligned}
& \qquad y \equiv 119 \beta^{2}-3 \alpha^{2}>84 \alpha \beta \\
& \text { i.e., }\left(\beta-\frac{42}{119} \alpha\right)^{2}>\frac{2359}{119^{2}} \alpha^{2} \\
& \text { i.e., } \beta>\frac{42+\sqrt{2359}}{119} \alpha=0.7610885 \alpha .
\end{aligned}
$$

With $\alpha=1, \beta=1$ in (4.5), one has $d=1$ and $y=114$, so that the resulting series is

$$
30^{2}+31^{2}+\ldots+198^{2}=(13 \times 124)^{2} .
$$

Letting $\alpha=1, \beta=2$ (so that $d=2, y=471$ ), one gets the series

$$
303^{2}+305^{2}+\ldots+639^{2}=(13 \times 481)^{2} .
$$

Again, letting $\alpha=2, \beta=3$, the series obtained is

$$
547^{2}+553^{2}+\ldots+2122^{2}=(13 \times 1091)^{2} .
$$

When $\alpha=5$, the minimum possible value of $\beta$ is $\beta=4$, and the corresponding series is

$$
99^{2}+119^{2}+\ldots+3459^{2}=(13 \times 2029)^{2} .
$$

When $\alpha=10$, the minimum admissible value of $\beta$ is $\beta=8 . \alpha=10, \beta=9$ gives the series

$$
1579^{2}+1669^{2}+\ldots+16699^{2}=(13 \times 10139)^{2} .
$$

Note that, one may get primitive solutions of the Diophantine equation (4.5) even when the parameters $\alpha$ and $\beta$ both are odd. 
By Lemma 2.4, the second solution is

$$
y=85 \beta^{2}-7 \alpha^{2}, d=\alpha \beta,
$$

where $\alpha$ and $\beta$ are integers with $\operatorname{gcd}(\alpha, \beta)=1, \alpha$ is not a multiple of 5 or $17, \beta$ is not a multiple of 7 , and $y \equiv 543 \beta^{2}-5 \alpha^{2}>84 d=84 \alpha \beta$. Then,

$$
\left(\beta-\frac{42}{85} \alpha\right)^{2}>\frac{2359}{85^{2}} \alpha^{2}
$$

i.e., $\beta>\frac{42+\sqrt{2359}}{85} \alpha=1.0655239 \alpha$.

When $\alpha=1$, the minimum $\beta$ is $\beta=2$, and the corresponding series is

$$
165^{2}+167^{2}+\ldots+501^{2}=(13 \times 347)^{2} .
$$

When $\alpha=2, \beta=3$ (so that $d=6, y=737$ ), the resulting series is

$$
233^{2}+239^{2}+\ldots+1241^{2}=(13 \times 793)^{2} .
$$

In this case, in order to get primitive solutions of the Diophantine equation (4.6), it is not necessary that the parameters $\alpha$ and $\beta$ must be of opposite parity, as is required in Lemma 2.4. For example, $\alpha=1, \beta=3$ gives the series

$$
506^{2}+509^{2}+\ldots+1010^{2}=(13 \times 772)^{2} .
$$

The next solution to consider is

$$
y=35 \beta^{2}-17 \alpha^{2}, d=\alpha \beta,
$$

where $\alpha$ and $\beta$ are integers with $\operatorname{gcd}(\alpha, \beta)=1, \alpha$ is not a multiple of 5 or 7 , and $\beta$ is not a multiple of 17 ; moreover, $y>84 d=84 \alpha \beta$. The latter condition may be put in the following form :

$$
\left(\beta-\frac{42}{35} \alpha\right)^{2}>\frac{2359}{35^{2}} \alpha^{2}
$$

i.e., $\beta>\frac{42+\sqrt{2359}}{35} \alpha=2.5877 \alpha$.

When $\alpha=1$, the minimum $\beta$ is $\beta=3$. The corresponding series is

$$
46^{2}+49^{2}+\ldots+550^{2}=(13 \times 332)^{2} .
$$

When $\alpha=2$, the minimum $\beta$ is $\beta=7$, giving the series

$$
471^{2}+485^{2}+\ldots+2823^{2}=(13 \times 3362)^{2} .
$$

When $\alpha=3$, the minimum $\beta$ is $\beta=8$, and the corresponding series is

$$
71^{2}+95^{2}+\ldots+4103^{2}=(13 \times 2393)^{2} .
$$

while $\alpha=3, \beta=11$ gives the series

$$
1310^{2}+1343^{2}+\ldots+6854^{2}=(13 \times 4388)^{2} .
$$

The above example shows that, to get primitive solutions of the Diophantine equation (4.7), it is not necessary that the parameters $\alpha$ and $\beta$ must be of opposite parity.

Finally, the fourth solution

$$
y=595 \beta^{2}-\alpha^{2}, d=\alpha \beta,
$$

is considered, where $\alpha$ and $\beta$ are integers with gcd $(\alpha, \beta)=1, \alpha$ is not a multiple of 5 or 7 or 17 . Now,

$$
y \equiv 595 \beta^{2}-\alpha^{2}>84 \alpha \beta
$$

i.e., $\left(\beta-\frac{42}{595} \alpha\right)^{2}>\frac{2359}{595^{2}} \alpha^{2}$

i.e., $\beta>\frac{42+\sqrt{2359}}{595} \alpha=0.1522177 \alpha$.

Thus, in this case, $\alpha$ and $\beta$ must satisfy the above inequality.

When $\alpha=1, \beta=1$, the corresponding series is

$$
510^{2}+511^{2}+\ldots+678^{2}=(13 \times 596)^{2} .
$$

With $\alpha=1, \beta=2$, the resulting series is

$$
2211^{2}+2213^{2}+\ldots+2547^{2}=(13 \times 2381)^{2},
$$

and with $\alpha=1, \beta=3$, the series obtained is

$$
5102^{2}+5105^{2}+\ldots+5606^{2}=(13 \times 5356)^{2} .
$$

When $\alpha=2, \beta=1$, the series obtained is

$$
423^{2}+425^{2}+\ldots+759^{2}=(13 \times 599)^{2} .
$$

Note that, when $\beta=1$, the maximum possible value of $\alpha$ is $\alpha=6$. Corresponding to $\alpha=6, \beta=1$, the series is

$$
55^{2}+61^{2}+\ldots+1063^{2}=(13 \times 6031)^{2} .
$$

Also, note that, primitive solutions of the Diophantine equation (4.8) are obtained even when the parameters $\alpha$ and $\beta$ are odd.

Example 4.5: (3.2) with $N=264$ gives

$$
S S(264)=23^{2}\left[y^{2}+5830(2 d)^{2}\right] \text {. }
$$

Assuming that $S S(264)=(23 x)^{2}$ for some integer $x$ (> 0 ), by Lemma 2.5 with $p=2, q=5, r=11, s=53$, one solution is

$$
y=2915 \beta^{2}-2 \alpha^{2}, d=\alpha \beta,
$$


where $\alpha$ and $\beta$ are integers with $\operatorname{gcd}(\alpha, \beta)=1, \alpha$ is not a multiple of 5 or 11 or 53 , and $\beta$ is not even. Now, the condition $y \equiv 2915 \beta^{2}-2 \alpha^{2}>264 d=264 \alpha \beta$ can be written as

$$
\begin{gathered}
\left(\beta-\frac{132}{2915} \alpha\right)^{2}>\frac{23254}{2915^{2}} \alpha^{2} \\
\text { i.e., } \beta>\frac{132+\sqrt{23254}}{2915} \alpha=0.097596 \alpha \text {. }
\end{gathered}
$$

Thus, in this case, $\alpha$ and $\beta$ must satisfy the condition above.

When $\alpha=1, \beta=1$ in (4.9), the series obtained is

$$
2649^{2}+2650^{2}+\ldots+3177^{2}=(23 \times 2917)^{2},
$$

and when $\alpha=2, \beta=1$, the series is

$$
2379^{2}+2381^{2}+\ldots+3435^{2}=(23 \times 2923)^{2} .
$$

Note that, when $\beta=1$, the maximum attainable $\alpha$ is $\alpha=10$. The case $\alpha=10, \beta=1$ is covered by the fifth solution considered later.

By Lemma 2.5, the second solution is

$$
y=1166 \beta^{2}-5 \alpha^{2}, d=\alpha \beta,
$$

where $\alpha$ and $\beta$ are integers with $\operatorname{gcd}(\alpha, \beta)=1, \alpha$ is not even or a multiple of 11 or 53 , and $\beta$ is not a multiple of 5 . Now,

$$
\begin{gathered}
y \equiv 1166 \beta^{2}-5 \alpha^{2}>264 \alpha \beta \\
\text { i.e., }\left(\beta-\frac{132}{1166} \alpha\right)^{2}>\frac{23254}{1166^{2}} \alpha^{2} \\
\text { i.e., } \beta>\frac{132+\sqrt{23254}}{1166} \alpha=0.24399 \alpha,
\end{gathered}
$$

which is the condition that must be satisfied.

When $\alpha=1, \beta=1$ in (4.10), the series is

$$
897^{2}+898^{2}+\ldots+1425^{2}=(23 \times 1171)^{2} .
$$

When $\alpha=3, \beta=1$, the corresponding series is

$$
329^{2}+332^{2}+\ldots+1913^{2}=(23 \times 1211)^{2} .
$$

Note that, when $\alpha=4$, the minimum admissible $\beta$ is $\beta=1$.

The third solution is

$$
y=530 \beta^{2}-11 \alpha^{2}, d=\alpha \beta,
$$

where $\alpha$ and $\beta$ are integers with $\operatorname{gcd}(\alpha, \beta)=1, \alpha$ is not even or a multiple of 11 , and $\beta$ is not a multiple of 11 . Now, the condition $y \equiv 530 \beta^{2}-11 \alpha^{2}>264 \alpha \beta$ may be simplified as follows :

$$
\left(\beta-\frac{132}{530} \alpha\right)^{2}>\frac{23254}{530^{2}} \alpha^{2}
$$

i.e., $\beta>\frac{132+\sqrt{23254}}{530} \alpha=0.5367785 \alpha$.

Thus, in this case, $\alpha$ and $\beta$ must satisfy the above condition.

When $\alpha=1, \beta=1$ in (4.11), the series is

$$
255^{2}+256^{2}+\ldots+783^{2}=(23 \times 541)^{2},
$$

and when $\alpha=1, \beta=2$, the series obtained is

$$
1581^{2}+1583^{2}+\ldots+2637^{2}=(23 \times 2131)^{2} .
$$

The solution to consider next is

$$
y=110 \beta^{2}-53 \alpha^{2}, d=\alpha \beta,
$$

where $\alpha$ and $\beta$ are integers with $\operatorname{gcd}(\alpha, \beta)=1, \alpha$ is not even or a multiple of 5 or 11 , and $\beta$ is not a multiple of 53. Now, $\alpha$ and $\beta$ satisfy the condition

$$
y \equiv 110 \beta^{2}-53 \alpha^{2}>264 \alpha \beta
$$

i.e., $\left(\beta-\frac{132}{110} \alpha\right)^{2}>\frac{23254}{110^{2}} \alpha^{2}$

i.e., $\beta>\frac{132+\sqrt{23254}}{110} \alpha=2.5862965 \alpha$.

When $\alpha=1$, the minimum attainable $\beta$ is $\beta=3$, giving the series

$$
145^{2}+148^{2}+\ldots+1729^{2}=(23 \times 1043)^{2} .
$$

When $\alpha=3$, the minimum admissible $\beta$ is $\beta=8$, and the corresponding series is

$$
227^{2}+251^{2}+\ldots+12899^{2}=(23 \times 7517)^{2} .
$$

Now, the fifth solution

$$
y=583 \beta^{2}-10 \alpha^{2}, d=\alpha \beta,
$$

is considered, where $\alpha$ and $\beta$ are integers with $\operatorname{gcd}(\alpha$, $\beta)=1, \alpha$ is not a multiple of 11 or 53 , and $\beta$ is not even or a multiple of 5 . Now, the condition $y \equiv 583 \beta^{2}$ $-10 \alpha^{2}>264 \alpha \beta$ can be written as

$$
\left(\beta-\frac{132}{583} \alpha\right)^{2}>\frac{23254}{583^{2}} \alpha^{2}
$$

i.e., $\beta>\frac{132+\sqrt{23254}}{583} \alpha=0.48798 \alpha$.

When $\alpha=1, \beta=1$ in (4.3), the series is

$$
309^{2}+310^{2}+\ldots+837^{2}=(23 \times 593)^{2},
$$

when $\alpha=2, \beta=1$, the resulting series is

$$
15^{2}+17^{2}+\ldots+1071^{2}=(23 \times 623)^{2} \text {. }
$$

Considering the solution

$$
y=265 \beta^{2}-22 \alpha^{2}, d=\alpha \beta,
$$




$$
y \equiv 265 \beta^{2}-22 \alpha^{2}>264 \alpha \beta
$$

i.e., $\left(\beta-\frac{132}{265} \alpha\right)^{2}>\frac{23254}{265^{2}} \alpha^{2}$

i.e., $\beta>\frac{132+\sqrt{23254}}{265} \alpha=1.073557 \alpha$.

When $\alpha=1$, the minimum admissible value of $\beta$ is $\beta$ $=3$, and the corresponding series is

$$
1571^{2}+1574^{2}+\ldots+3155^{2}=(23 \times 2407)^{2} .
$$

When $\alpha=2$, the minimum admissible $\beta$ is $\beta=3$, and the corresponding series is

$$
713^{2}+719^{2}+\ldots+3881^{2}=(23 \times 2473)^{2} .
$$

Next, the seventh solution

$$
y=106 \beta^{2}-55 \alpha^{2}, d=\alpha \beta,
$$

is considered, where $\alpha$ and $\beta$ are integers with $\operatorname{gcd}(\alpha$, $\beta)=1, \alpha$ is not even or a multiple of 53 , and $\beta$ is not multiple of 5 or 11 . Now, the condition $y \equiv 106 \beta^{2}-$ $55 \alpha^{2}>264 \alpha \beta$ can be written as

$$
\left(\beta-\frac{132}{106} \alpha\right)^{2}>\frac{23254}{106^{2}} \alpha^{2}
$$

i.e., $\beta>\frac{132+\sqrt{23254}}{106} \alpha=2.6838926 \alpha$.

When $\alpha=1$, the minimum possible $\beta$ is $\beta=3$, giving the series

$$
107^{2}+110^{2}+\ldots+1691^{2}=(23 \times 1009)^{2} .
$$

Finally, the solution to consider is

$$
y=5830 \beta^{2}-\alpha^{2}, d=\alpha \beta,
$$

where $\alpha$ and $\beta$ are integers with $\operatorname{gcd}(\alpha, \beta)=1, \alpha$ is not even or a multiple of 5 or 11 or 53 . Here,

$$
\begin{gathered}
y \equiv 5830 \beta^{2}-\alpha^{2}>264 \alpha \beta \\
\text { i.e., }\left(\beta-\frac{132}{5830} \alpha\right)^{2}>\frac{23254}{5830^{2}} \alpha^{2} \\
\text { i.e., } \beta>\frac{132+\sqrt{23254}}{5830} \alpha=0.048798 \alpha \text {. }
\end{gathered}
$$

Thus, in this case, $\alpha$ and $\beta$ must satisfy the inequality above.

When $\alpha=1, \beta=1$ in (4.16), the series is

$$
5565^{2}+5566^{2}+\ldots+6093^{2}=(23 \times 5831)^{2},
$$

and when $\alpha=1, \beta=2$, the series is

$$
22791^{2}+22793^{2}+\ldots+23847^{2}=(23 \times 23321)^{2} .
$$

\section{Some remarks}

This papers finds a set of necessary conditions. The sum of squares of $(2 N+1)$ positive integers in the arithmetic progression is a perfect square under two simple and plausible assumptions. It is shown that the problem gives rise to a Diophantine equation. The problem is then illustrated with five examples. These examples show that, for any $N$ fixed, there is, in fact, an infinite number of solutions. There is an infinite number of instances such that the sum of squares of length $(2 N+1)$ is a perfect square. In this connection, the following result holds true.

Lemma 5.1: Two solutions of the equati $2 N+1=M^{2}$ are as follows :

(1) $N=6 a(3 a-1)$ for any integer $a \geq 1$,

(2) $N=6 b(3 b+1)$ for any integer $b \geq 1$.

Proof: By Lemma 3.2, any integer $N$ satisfying the given equation must form $N=12 n$, for some integer $n$. Then, the equation takes the form

$$
24 n=(M-1)(M+1) \text {. }
$$

Now, one of the following two cases may result :

Case 1: $M-1=4 m$ for some integer $m \geq 1$.

In this case, from (5.1),

$$
3 n=m(2 m+1) \text {. }
$$

Then, there are two possibilities; namely, one of the two factors on the r.h.s. is divisible by 3. First, let $2 m$ $+1=3 a$ for some integer $a \geq 1$. Substituting in (5.2), one gets

$$
n=a\left(\frac{3 a-1}{2}\right)
$$

so that

$$
N=6 a(3 a-1) \text {. }
$$

Next, let $m=3 x$ for some integer $x \geq 1$. Then, (5.2) gives $n=x(6 x+1)$, so that $N=12 x(6 x+1)$.

Case 2: $M-1=4 m-2$ for some integer $m \geq 1$.

Here, from (5.1),

$$
3 n=m(2 m-1) \text {. }
$$

Then, one of the two factors on the r.h.s. is divisible by 3 . Letting $2 m-1=3 b$ for some integer $b \geq 1$, (5.2) gives

$$
n=b\left(\frac{3 b+1}{2}\right)
$$

so that

$$
N=6 b(3 b+1) \text {. }
$$


Next, letting $m=3 y(y \geq 1$ being an integer) in the equation (5.4), one gets $n=y(6 y-1)$, so that $N=$ $12 y(6 y-1)$, which is a particular case of (5.3).

A related problem was proposed by Alfred (1964), which was later revived by Laub (1990). The problem is to find the conditions such that the sum of squares of consecutive integers is a perfect square. The problem has been treated by Beeckmans (1994) and Hirschhorn (2011). In this paper, formulation of the problem treated the above problem corresponds to the particular case when $d=1$ (corresponding to $\alpha=1$, $\beta=1$ ). In Example 4.1, letting $\alpha=1, \beta=1$, the following series in arithmetic progression is obtained :

$$
1^{2}+2^{2}+\ldots+24^{2}=(5 \times 14)^{2} .
$$

This result is interesting. Examples $4.1-4.5$ together prove that the sum of squares of the first $(2 N+1)$ natural numbers is not a perfect square when $N=12,24,60,84$, 264. Example 4.2 reveals the interesting result that

$$
25^{2}+26^{2}+\ldots+73^{2}=(7 \times 51)^{2} .
$$

Thus, the sum of squares of the first 24 natural numbers is a perfect square, and the sum of squares of the next 49 natural numbers is also a perfect square.

It may be proved that, for any integer $N(>0)$, there is a series of sum of squares of $(2 N+1)$ consecutive natural numbers (satisfying both Assumption 3.1 and Assumption 3.2), which is a perfect square. The proof is as follows : Letting $N=12 n, S S(N)$, given by (3.2), may be cast as follows :

$$
\begin{aligned}
& S S(12 n) \\
& =(24 n+1)\left[y^{2}+n(12 n+1)(2 d)^{2}\right], y>12 n d,
\end{aligned}
$$

where

$$
y=n(12 n+1) \beta^{2}-\alpha^{2}, d=\alpha \beta .
$$

It now remains to show that, $y>12$ nd when $\alpha=1$, $\beta=1$, that is,

$$
n(12 n+1)-1>12 n,
$$

that is,

$$
f(n) \equiv 12 n^{2}-11 n-1>0 .
$$

Since $f(n)$ is strictly increasing in $n$, it follows that $f(n)>f(1)=0$ for all $n \geq 2$. The series in (5.6) proves the case when $n=1$.

Another problem of interest, initiated by Alfred (1967), is the problem of finding conditions such that the sum of squares of consecutive odd integers is a perfect square. Note that, this problem corresponds to $d=2$ with $y-N d$ being odd. It can be shown that, for any integer $N(>1)$ (satisfying Assumption 3.1 and Assumption 3.2), there always exists a sequence of consecutive odd natural numbers whose sum of squares is a perfect square. This can be done as follows : If $n$ is odd, choose $\quad \alpha=1, \beta=2$ in (5.8), otherwise, choose $\alpha=2, \beta=1$.

\section{Conflicts of Interest}

The author declare that this is no conflicts of interest, financial or otherwise.

\section{References}

Alfred U. Consecutive integers whose sum of squares is a perfect square. Math. Mag. 1964; 37(1): 19-32.

Alfred U. Sum of squares of consecutive odd integers. Math. Mag. 1967; 40(4): 194-199.

Beeckmans L. Squares expressible as sum of consecutive squares. Amer. Math. Monthly, 1994; 101(5): 437-442.

Dickson LE. History of the Theory of Numbers, Vol. II, Chelsea, N.Y., U.S.A. 1971.

Hirschhorn MD. When is the sum of consecutive squares a square. Math. Mag. 2011; 95(534): 511-512.

Laub M. Advanced Problem 6552: Squares expressible as a sum of $\mathrm{n}$ consecutive squares. Amer. Math. Monthly, 1990; 97: 622-625.

Majumdar AAK. A note on the Diophantine equation $\mathrm{x}^{2}=\mathrm{y}^{2}+3 \mathrm{z}^{2}$. J. Bangladesh Acad. Sci., 2020; 44(2): 201-205 (Short Communication).

Majumdar AAK. On the Diophantine equation $\mathrm{x}^{2}=\mathrm{y}^{2}+3 \mathrm{z}^{2}$. Jahangirnagar J. Math. Math. Sci., 2018; 31: 1-8.

Sastry KRS. Problems and Solutions. Problem No. 469: Arithmetic progression of length 49 and square sums of squares solution. Coll. Math. J. 1992; 23(1): 70.

Vowe M. Problems and Solutions. Solution to Problem No. 469: A sum of 49 squares. Coll. Math. J. 1993; 24(1): 99. 